\title{
Low-dose nanotherapy-mediated site-specific inhibition of neutrophil extracellular traps for immunoregulatory treatment of asthma
}

\section{Yongchang $\mathrm{Ma}$}

Third Military Medical University

Lanlan Li

Third Military Medical University

Yi Hu

Third Military Medical University

Songling Han

Third Military Medical University

Qinghua Yang

Third Military Medical University

Wendan Pu

Third Military Medical University

Xiangjun Zhang

Third Military Medical University

Jianxiang Zhang ( $\nabla$ jxzhang1980@gmail.com )

Third Military Medical University

Article

Keywords: Asthma, Neutrophil extracellular traps, TCPN

Posted Date: February 26th, 2021

DOl: https://doi.org/10.21203/rs.3.rs-200054/v1

License: (c) (1) This work is licensed under a Creative Commons Attribution 4.0 International License.

Read Full License 


\section{Abstract}

Asthma, one of the most common lung diseases, remains a serious global healthy problem. Currently there is still an urgent need for effective and safe therapies against severe asthma. Neutrophil extracellular traps (NETs) have emerged as a new therapeutic target for different diseases, while precision regulation of NETs is highly challenging. Here, we report site-specific attenuation of oxidative stress in lung neutrophils via a cyclic oligosaccharide-derived nanotherapy (termed as TPCN) effectively inhibited the development of mouse neutrophilic asthma, a typical phenotype of severe asthma. Therapeutically, TPCN delivered via either intravenous injection or inhalation can distribute in lung neutrophils of asthmatic mice, thereby significantly mitigating oxidative stress, suppressing inflammatory responses, reversing airway remodelling, and improving pulmonary function. Notably, TPCN is effective even at an actual inhalation dose as low as $0.063 \mathrm{mg} / \mathrm{kg}$. Mechanistically, therapeutic benefits of TPCN are achieved by inhibiting reactive oxygen species (ROS)-induced formation of NETs, which further promotes immune homeostasis via regulating balance between regulatory $T$ (Treg) and T helper 17 (Th17) cells. Thus, TPCN holds great promise for precision therapy of neutrophilic asthma, while the ROS-NETsTreg/Th17 pathway can function as intriguing therapeutic targets for the treatment of severe asthma and other neutrophil-mediated inflammatory diseases.

\section{Background}

Asthma, characterized by airway inflammation and structural remodelling, is the second most prevalent chronic lung disease worldwide ${ }^{1-3}$. People affected by asthma increased by $29.4 \%$ from about 211 million in 1990 to more than 272 million in $2017^{3}$, leading to a major public health problem with a substantial socioeconomic burden. Drugs including corticosteroids, long-acting $\beta 2$-agonists and muscarinic antagonists, leukotriene receptor antagonists, and biologic therapies have been clinically used for asthma therapy ${ }^{1,4,5}$. In particular, inhalation of corticosteroids is the most frequently employed strategy for the management of asthma. However, long-term use of corticosteroids is generally accompanied with multiple adverse effects, such as adrenal suppression, osteoporosis, and glaucoma ${ }^{6,7}$. Moreover, high doses of oral or inhaled corticosteroids can result in airway neutrophilia, mainly by inducing recruitment and inhibiting apoptosis of neutrophils in the airways ${ }^{8-10}$. Notably, severe asthma, characterized by neutrophilic inflammation, responds poorly to corticosteroids and shows insufficient benefits after standard treatment $4,11,12$. Candidate therapies for neutrophilic asthma, such as macrolides and antagonists/antibodies to key pro-inflammatory cytokines, pose safety issues and unfavourable riskbenefit profiles ${ }^{4,11}$. Consequently, there is an unmet need for new therapeutic strategies for severe asthma. In addition, personalized approaches for both prevention and cure of poorly controlled asthma are still required ${ }^{1}$.

Recent studies have revealed critical roles of neutrophils in the pathogenesis of asthma. In addition to mediating severe asthma, neutrophils have crucial roles in type 2 allergic asthma induced by lipopolysaccharide, viruses, or ozone $\mathrm{e}^{13-15}$, by releasing neutrophil extracellular traps (NETs) that are web- 
like extracellular structures of chromatin filaments coated with histones, proteases, and cytosolic/granule proteins $^{16}$. Although NETosis, known as the formation of NETs by neutrophils, can prevent dissemination of different pathogens by immobilizing and catching them, dysregulated NETs are responsible for the pathogenesis of numerous inflammatory and immune diseases ${ }^{16,17}$. Therefore, inhibiting NETosis or eliminating excessive NETs has been implicated as promising therapeutic targets for different diseases varying from autoimmune diseases to infectious and non-infectious diseases ${ }^{17,18}$. However, it remains unclear whether this NETosis inhibition strategy is efficacious for the treatment of asthma, in particular neutrophilic asthma.

Here we report that site-specific inhibition of NETosis in the lungs can effectively suppress local inflammation, reduce airway remodelling, and improve lung function, thereby alleviating neutrophilic asthma in mice, which was achieved by selectively attenuating oxidative stress via either intravenous or inhaled nanotherapies (Fig. 1a). Furthermore, the suppressed NETs formation also afforded beneficial effects on the balance of T helper 17 (Th17) and regulatory T (Treg) cells by reducing NET trapping of naïve $T$ cells. Thus, targeted inhibition of NETosis in the lungs via antioxidant nanotherapies represents a promising, translational, and pathway-specific strategy for the prevention and treatment of severe asthma.

\section{Preparation and characterization of a bioactive nanotherapy}

A reactive oxygen species (ROS)-scavenging bioactive material (i.e., TPCD) was synthesized by covalently conjugating 4-hydroxy-2,2,6,6-tetramethylpiperidin-1-oxyl (Tempol) and 4(hydroxymethyl)phenylboronic acid pinacol ester (PBAP) onto $\beta$-cyclodextrin $(\beta$-CD), a cyclic oligosaccharide ${ }^{19}$. The chemical structure of TPCD was confirmed by Fourier Transform infrared and ${ }^{1} \mathrm{H}$ NMR spectroscopy (Supplementary Fig. 1). Calculation according to the ${ }^{1} \mathrm{H}$ NMR spectrum revealed 2 Tempol and 5 PBAP units in each TPCD. Then TPCD nanoparticles (defined as TPCN) were produced by a nanoprecipitation/self-assembly method (Fig. 1b). Thus obtained TPCN displayed well-defined spherical shape, as illustrated by transmission electron microscopy (TEM) and scanning electron microscopy (SEM) images (Fig. 1c-d), with a narrow size distribution and a mean diameter of $80 \mathrm{~nm}$ (Fig. 1e).

\section{Site-specific delivery of TPCN to pulmonary neutrophils via intravenous injection}

A mouse model of neutrophilic asthma was established by stimulation with ovalbumin (OVA) and $\mathrm{Al}(\mathrm{OH})_{3}$ via intraperitoneal (i.p.) injection, in combination with intranasal (i.n.) administration of lipopolysaccharide (LPS) and OVA (Fig. 2a) ${ }^{20}$. Subsequently, in vivo targeting capability of TPCN was examined, using Cy7.5-labeled TPCN (Cy7.5/TPCN) (Fig. 2a, the upper panel). At $24 \mathrm{~h}$ after intravenous (i.v.) injection, ex vivo imaging revealed notable Cy7.5 fluorescence in the lungs of mice with induced asthma (Fig. 2b). Of note, asthmatic mice displayed significantly higher pulmonary accumulation of Cy7.5/TPCN than that of normal animals (Supplementary Fig. 2). This passive targeting was mainly attributed to the structurally and functionally abnormal respiratory epithelium in asthma ${ }^{21}$, which may cause barrier dysfunction and epithelial permeability to different molecular and particulate substances ${ }^{22-}$ 
24 . In a separate study, flow cytometry showed $92 \pm 3 \%$ Cy 5 -positive neutrophils in pulmonary tissues at 24 h after i.v. injection of Cy5-labeled TPCN (Fig. 2c). Further immunofluorescence analysis revealed considerable fluorescence co-localization of Cy5 and Ly6G (Fig. 2d), a typical neutrophil biomarker. Collectively, these results demonstrated that i.v. delivered TPCN can site-specifically distribute in pulmonary neutrophils of asthmatic mice.

\section{Targeted therapy of asthma by i.v. delivery of TPCN}

Then we evaluated in vivo efficacy of TPCN in asthmatic mice (Fig. 2a, the lower panel). We found that i.v. treatment with different doses of TPCN notably decreased the ROS level in bronchoalveolar lavage fluid (BALF) collected from diseased mice (Fig. 2e). Also, TPCN effectively reduced typical proinflammatory cytokines, including tumor necrosis factor (TNF)-a, interleukin (IL)-1 $\beta$, and IL-17 (Fig. 2f-h), which are implicated in the pathogenesis of asthma ${ }^{25}$. Consistently, we detected notable decrease in the myeloperoxidase (MPO) level and neutrophil count in BALF and lungs after therapy with TPCN (Fig. 2i-j and Supplementary Fig. 3), while their high expression is a common feature of the OVA/LPS-sensitized model of neutrophilic asthma ${ }^{20}$. Further inspection on histological sections of intrapulmonary bronchi stained with either hematoxylin and eosin (H\&E) or periodic acid Schiff (PAS) revealed sloughing of epithelial cells, reticular basement membrane thickening, inflammatory cell infiltration in airway mucosa, enlargement of bronchial smooth muscle, and mucus hypersecretion for diseased mice (Fig. 2k-I). After therapy with TPCN, we observed a pseudostratified ciliated columnar epithelium, an indistinct reticular basement membrane, few inflammatory cells, and considerably reduced bronchial smooth muscle. Mice treated with $1 \mathrm{mg} / \mathrm{kg}$ TPCN displayed nearly unaltered microstructures, which are even comparable to the normal group. Further, pulmonary function was examined by a methacholine challenge test. Whereas asthmatic mice showed high airway response to increased doses of methacholine (Fig. $2 \mathrm{~m}$ ), treatment with $1 \mathrm{mg} / \mathrm{kg}$ TPCN completely attenuated pulmonary resistance $\left(R_{L}\right)$, similar to that of normal mice.

\section{Site-specific delivery of TPCN to lung neutrophils via aerosol inhalation}

Since inhalation therapy is the most preferred strategy for the treatment of pulmonary diseases, we assessed whether inhaled TPCN can be delivered to lung neutrophils. Asthmatic mice were induced as aforementioned (Fig. 3a, the upper panel), which were transiently accommodated in a chamber with aerosolized aqueous solution of TPCN (Fig. 3b). At $24 \mathrm{~h}$ after inhalation, effective accumulation of Cy7.5/TPCN in the lungs was observed by ex vivo imaging (Fig. 3c). A separate analysis by flow cytometry revealed $93 \pm 3 \%$ lung neutrophils were Cy5-positive at $24 \mathrm{~h}$ after inhalation of Cy5/TPCN in asthmatic mice (Fig. 3d). This neutrophil-specific distribution was confirmed by immunofluorescence analysis of lung cryosections (Fig. 3e). Consequently, TPCN can also be effectively delivered to pulmonary neutrophils by aerosol inhalation.

\section{Treatment of asthma by aerosol inhalation of TPCN}

Then therapeutic effects of inhaled TPCN were evaluated in asthmatic mice. In this case, budesonide (BDN), a commonly used drug, was employed as a positive control. All formulations were administered by 
inhalation after nebulization. Treatment with TPCN at theoretical doses of 0.1 and $1 \mathrm{mg} / \mathrm{kg}$, particularly the high dose, significantly reduced the levels of ROS, TNF- $\alpha$, IL-1 $\beta$, and IL-8 in BALF, compared to the model group administered saline (Fig. $3 f-i)$. Also, the MPO level and neutrophil count in BALF and pulmonary tissues notably decreased after TPCN inhalation (Fig. 3j-k and Supplementary Fig. 4). In particular, inhalation of TPCN at $1 \mathrm{mg} / \mathrm{kg}$ resulted in significantly lower levels of serum immunoglobulin $\mathrm{E}$ (IgE, an antibody that mediates allergic reactions and plays a critical role in allergic asthma) (Fig. 3l). Examination on H\&E or PAS sections showed notable attenuation of inflammatory cell infiltration, pulmonary edema, and mucous secretion after inhalation of $1 \mathrm{mg} / \mathrm{kg}$ TPCN (Fig. 3m-n). By contrast, except notably reduced MPO and neutrophil levels, inhalation of BDN at a clinically relevant dose afforded no significant benefits, with respect to attenuating oxidative stress and suppressing inflammation in lungs. This is consistent with the previous finding that neutrophilic asthma is generally steroid-resistant ${ }^{2}$. Correspondingly, TPCN inhalation at $1 \mathrm{mg} / \mathrm{kg}$ effectively improved pulmonary function (Fig. 3o). Accordingly, TPCN delivered by inhalation is also effective in the treatment of asthma. It should be noted that, the estimated pulmonary delivery efficiency of inhalation was $6.3 \%$, by comparing Cy 5 fluorescence intensities in lungs after direct pulmonary administration and aerosol inhalation (Supplementary Fig. 5). This suggested that TPCN is efficacious even at an actual inhaled dose of 0.063 $\mathrm{mg} / \mathrm{kg}$.

\section{Treatment of asthma in mice by a mitochondrial-targeting TPCN nanotherapy}

Since TPCN is expected to exert its efficacy by site-specifically eliminating oxidative stress in lung neutrophils, we further examined whether mitochondrial-targeting of TPCN can enhance its therapeutic effects. As a proof of concept, TPCD nanoparticles were prepared by nanoprecipitation/self-assembly in the presence of stearyl triphenylphosphonium ${ }^{26}$, giving rise to a mitochondrial-targeting nanotherapy (TTPCN). TEM and SEM observation revealed that TTPCN displayed well-defined spherical morphology and narrow size distribution (Fig. 4a-c), with the mean diameter of $105 \mathrm{~nm}$ and $\zeta$-potential of $-10.6 \mathrm{mV}$. Using Cy5-labeled TTPCN, confocal microscopic observation demonstrated excellent mitochondrialtargeting capability of TTPCN in neutrophils (Fig. 4d), which was also confirmed in human pulmonary epithelial cells (Supplementary Fig. 6).

Interestingly, Cy5/TTPCN showed significantly higher pulmonary accumulation than Cy5/TPCN, after i.v. delivery in asthmatic mice (Fig. 4e). Of note, a high neutrophil distribution in lung tissues was detected (Fig. 4f-g). Subsequently, therapeutic benefits of TTPCN were tested, following the similar i.v. treatment procedures (Fig. 2a, the lower panel). Compared to the same dose of TPCN $(1 \mathrm{mg} / \mathrm{kg})$, TTPCN more effectively inhibited the expression levels of ROS, TNF- $a$, IL-1 $\beta$, IL-8, and MPO as well as the neutrophil count in BALF (Fig. 4h-m). Inspection on histological sections stained with H\&E or PAS also revealed desirable efficacy of TTPCN (Fig. 4n-o). Consequently, surface decoration with a mitochondrial-targeting unit can simultaneously enhance pulmonary delivery efficiency and in vivo efficacy of TPCN.

\section{In vitro and in vivo mechanistic studies}


In vitro antioxidative stress and anti-inflammatory effects of TPCN in neutrophils. After in vitro incubation, microscopic observation and flow cytometric quantification showed effective endocytosis of Cy5/TPCN by mouse peritoneal neutrophils, in both time- and dose-dependent manners (Fig. 5a-b and Supplementary Fig. 7-8). Correspondingly, treatment with various doses of TPCN significantly inhibited phorbol 12-myristate 13-acetate (PMA)-induced ROS generation in neutrophils (Fig. 5c-d and Supplementary Fig. 9). Also, overexpression of typical pro-inflammatory cytokines (TNF-a, IL-1 $\beta$, and IL-8) in neutrophils was remarkably suppressed by TPCN (Fig. 5e-g). In addition, migration of neutrophils was significantly attenuated after TPCN treatment (Fig. 5h and Supplementary Fig. 10). Of note, the mitochondrial-targeting nanotherapy TTPCN more effectively reduced ROS production in neutrophils, as compared to TPCN (Supplementary Fig. 11). These results suggested that TPCN can markedly inhibit oxidative stress and inflammatory response in neutrophils after efficient cellular internalization, in line with in vivo results.

TPCN inhibits the formation of neutrophil extracellular traps. To further examine mechanisms underlying anti-asthmatic effects of TPCN, mRNA expression profiles of pulmonary tissues were identified by RNAsequencing. Among all detected mRNAs, there were 197 and 9 differentially up-regulated and downregulated genes in asthmatic mice (relative to healthy mice) and TPCN-treated asthmatic mice (relative to asthmatic mice), respectively (Supplementary Fig. 12). We identified 20 significantly up-regulated mRNAs in asthmatic mice compared to healthy mice (Fig. 5i). In particular, neutrophil-associated genes Elane and $M p o$ were expressed at significantly higher levels in asthmatic mice than those of healthy mice, which were recovered to the normal levels after TPCN therapy. qRT-PCR analysis confirmed the abnormal expression levels of these two candidate transcripts and significant inhibitory effects of TPCN (Fig. 5j-k). As well demonstrated, neutrophil elastase (NE) and MPO, separately encoded by Elane and Mpo genes, are the main components of NETs ${ }^{27,28}$. Therefore we hypothesize that the development of neutrophilic asthma is closely related to NETs, while therapeutic effects of TPCN are mainly mediated by inhibiting NETs formation.

As expected, expression levels of the characteristic components of NETs, such as extracellular doublestranded DNA (dsDNA), NE, MPO, and citrullinated histone $\mathrm{H} 3$ (citH3, a modified form of histone $\mathrm{H} 3$ implicated in chromatin decondensation and NETs formation) significantly increased in PMA-activated neutrophils (Fig. 6a-e), which were remarkably reduced by TPCN in a dose-response pattern. Further, in vitro NETs formation by PMA-stimulated neutrophils was directly observed by immunofluorescence, as illustrated by release of extracellular dsDNA and citH3 that appeared as fibrous strands (Fig. $6 \mathrm{f}$ and Supplementary Fig. 13). By contrast, TPCN treatment significantly reduced the degree of NETosis and citH3 area (Fig. 6f-h). These results substantiated that TPCN can effectively inhibit in vitro NETs formation.

Moreover, asthmatic mice showed significantly higher levels of dsDNA and NE in BALF as well as higher $\mathrm{NE}$ and citH3 in lungs, compared to normal mice (Fig. 6i-m). In addition, NETs formation in pulmonary tissues of asthmatic mice was affirmed by immunofluorescence analysis (Fig. 6n and Supplementary Fig. 14), as indicated by citH3 and NE positive fluorescence signals. Treatment with TPCN at either 0.1 or 
$1 \mathrm{mg} / \mathrm{kg}$ remarkably reduced expressions of dsDNA, NE, and citH3 in BALF and lungs. These results demonstrated that TPCN can attenuate NETs formation in the lungs of asthmatic mice.

TPCN regulates NETs-mediated Treg/Th17 cell imbalance. Previous studies have demonstrated that Treg cells play a crucial role in the maintenance of immune homeostasis in the airways ${ }^{29}$. Particularly, both animal and clinical studies indicated that Treg/Th17 cell imbalance considerably contributes to the pathogenesis of asthma ${ }^{30,31}$. To explore whether inhibition of NETs formation by TPCN and its antiasthmatic effects are related to Treg/Th17 homeostasis, we analyzed numbers of pulmonary and splenic Treg/Th17 cells. Compared with those of healthy mice, the proportion of pulmonary Treg cells in asthmatic mice significantly decreased, while an opposite change was observed for Th17 cells in lungs (Fig. 7a-d), resulting in a remarkably decreased ratio of Treg/Th17 cells (Fig. 7e). Similar changing profiles were observed for the numbers of splenic Treg and Th17 cells as well as their ratios (Fig. $7 f-j$ ). By contrast, treatment with TPCN significantly increased Treg cells and decreased Th17 cells, and therefore effectively reversed the proportion of Treg cells in both lungs and spleens. These data substantiated that TPCN can regulate the Treg/Th17 balance in asthmatic mice.

Since recent studies have revealed an important role of neutrophils and NETosis in mediating inflammatory and immune responses in asthma ${ }^{13,14}$, we speculate that Treg/Th17 imbalance is induced by NETs. Our in vitro studies indicated that NETs notably suppressed the differentiation of naïve T cells into Treg cells, whereas normal neutrophils showed no significant effects (Fig. 7k-I). Further SEM observation revealed that naïve T cells could be covered by NETs (Fig. $7 \mathrm{~m}$, left). Accordingly, NETsinhibited Treg differentiation might be resulted from the NETs coating-derived physical barriers on naïve T cells that may impair effective diffusion of Treg-inducing molecules and their close contact with $T$ cells. Treatment with TPCN significantly restored Treg differentiation from naïve T cells in the presence of NETs (Fig. 7n-o). Consistently, the coverage of T cells by NETs considerably decreased after TPCN treatment (Fig. $7 \mathrm{~m}$, right). In this case, only a small proportion of T cells were trapped by NETs.

\section{Safety studies on TPCN after inhalation}

Because TPCN is expected to be administered by aerosol inhalation, acute toxicity evaluation was performed in mice after nebulized inhalation at 50 or $100 \mathrm{mg} / \mathrm{kg}$ for 7 consecutive days (Supplementary Fig. 15a). During treatment, all treated mice remained healthy, without any behavioral abnormalities. In addition, all animals displayed similar change profiles in body weight gain and showed comparable organ indices for major organs at day 15 after treatment (Supplementary Fig. 15b-c). Examination on H\&E-stained sections of major organs revealed no discernible destruction of tissue microstructure or inflammatory cell infiltration in TPCN groups (Supplementary Fig. 16). Moreover, TPCN treatment did not lead to significant changes of oxidative stress-related factors and pro-inflammatory cytokines in lung tissues, such as $\mathrm{H}_{2} \mathrm{O}_{2}, \mathrm{MPO}$, TNF- $\mathrm{a}$, and IL-1 $\beta$ (Supplementary Fig. 17a-d). Likewise, there were no abnormal changes in typical hematological parameters and biomarkers relevant to liver/kidney functions in TPCN-treated mice (Supplementary Fig. 17e-l). In line with these results, we detected efficient hydrolysis and/or metabolism of TPCN in neutrophils and mouse lung homogenates (Supplementary Fig. 
18), resulting in parent $\beta-C D$ and other water-soluble molecules that can be cleared by the kidneys. Collectively, these preliminary data revealed good safety of TPCN after inhalation at a dose that is 100fold higher than those examined in therapeutic studies.

\section{Discussion}

Approximately $3-10 \%$ of people with asthma suffer from severe asthma ${ }^{5,32}$. This debilitating and treatment refractory disease places a large physical, psychological, social, and economic burden on patients. In addition to other daily medications, high doses of inhaled corticosteroids or long-term oral corticosteroids are generally necessary for the treatment of severe asthma. However, long-term corticosteroids frequently cause serious medication side-effects, such as obesity, diabetes, osteoporosis, hypertension, adrenal suppression, and depression/anxiety 6,7 . Add-on treatments for severe asthma, including tiotropium, leukotriene receptor antagonist, macrolides, and biologic agents, also show varied degrees of limitations. In particular, the inconvenience and high cost of long-term administration, variation in responses, and possible severe allergic reactions are major concerns of biologics used for severe asthma ${ }^{33,34}$. Consequently, great challenges remain in the treatment of severe asthma.

About half of patients with severe asthma have neutrophilic inflammation ${ }^{14}$, and airway neutrophilia is closely associated with asthma severity ${ }^{11}$. NETs released by activated neutrophils show important roles in the pathogenesis of severe asthma and other asthma phenotypes ${ }^{13-16,35,36}$. These findings implied that NETs can serve as a promising therapeutic target for asthma. Indeed, blocking NETosis by inhibitors of NE and protein-arginine deiminase 4 or degrading NETs with DNase can attenuate airway hyperresponsiveness ${ }^{13-15}$. However, currently existing molecules used for NETosis inhibition display real problems, such as specificity and safety concerns ${ }^{4,17,18}$. Here we found that an antioxidative and antiinflammatory nanotherapy TPCN can notably accumulate in the inflamed lungs of mice with neutrophilic asthma, after either i.v. or inhalation administration. The enhanced accumulation of TPCN in lung tissues is mainly due to the abnormal structure and function of the respiratory epithelium in asthmatic mice ${ }^{21,22}$ Of note, i.v. delivered or inhaled TPCN was mainly distributed in lung neutrophils, showing above $90 \%$ TPCN-positive neutrophils. Accordingly, the nanotherapy TPCN can be site-specifically delivered to lung neutrophils. Consistently, treatment with TPCN via either i.v. injection or inhalation in asthmatic mice afforded beneficial therapeutic effects, as manifested by significantly decreased oxidative stress, attenuated inflammatory reactions, inhibited mucus hypersecretion, suppressed airway remodeling, and improved airway hyperresponsiveness. Notably, bronchial hyperresponsiveness of asthmatic mice was almost completely reversed after TPCN therapy. Compared to BDN, a commonly used drug for asthma, inhaled TPCN more effectively alleviated asthma symptoms. Moreover, surface functionalization of TPCN with a mitochondrial-targeting moiety further improved its antioxidative activity and lung accumulation efficiency, thereby leading to additionally potentiated anti-asthmatic efficacies in mice. Collectively, the nanotherapy TPCN can effectively improve asthma symptoms, lung function, and airway hyperresponsiveness, by site-specifically attenuating oxidative stress in lung neutrophils. It is worth noting that TPCN is efficacious at very low doses (especially inhalation at $0.063 \mathrm{mg} / \mathrm{kg}$ ), which are 
dramatically lower than those of currently used drugs (varying from 0.26 to $78 \mathrm{mg} / \mathrm{kg}$ ). In addition, preliminary in vivo tests in mice suggested that TPCN was safe after 7-day continuous inhalation at a dose 100 -fold higher than those used in therapeutic assessment.

In line with in vivo efficacies, TPCN effectively inhibited oxidative stress and inflammatory responses in neutrophils in vitro. RNA-sequencing analysis of lung tissues revealed that TPCN treatment normalized abnormally up-regulated genes relevant to NETs, agreeing with the previous finding that oxidative stress has been implicated in the formation and release of $\mathrm{NETs}^{37,38}$. Furthermore, both in vitro and in vivo studies demonstrated that TPCN significantly inhibited the formation of NETs. These results substantiated that TPCN can site-specifically block NETosis by eliminating ROS in lung neutrophils.

On the other hand, increasing evidence has revealed the intimate relationship between immune dysfunction and asthma ${ }^{1}$. Th17 cells are associated with the neutrophilic inflammation and more severe phenotypes of asthma ${ }^{39}, 40$, while Treg cells display indispensable effects in anti-inflammation, maintaining self-tolerance, and preventing allergic diseases ${ }^{41}$. Also, previous studies indicated that Treg cells may interfere with asthma at different stages, such as sensitization, allergic inflammation, airway remodeling, and airway hyperresponsiveness ${ }^{42,43}$. Our results showed that asthmatic mice exhibited significantly increased Th17 cells in the spleen and lungs as compared to healthy mice, concomitant with notably decreased Treg cells and a reduced Treg/Th17 cell ratio. By contrast, treatment with TPCN normalized Treg and Th17 cell counts and their ratios to the levels comparable to those of healthy mice. This finding is consistent with the fact that Treg/Th17 cell imbalance plays a pivotal role in the development, exacerbation, and severity of asthma, especially in neutrophilic asthma ${ }^{30,31,44-46}$. We further found that TPCN can rescue NET-attenuated Treg cell differentiation from naïve $T$ cells, probably by decreasing NETs coating on T cells and inhibiting detrimental effects of the DNA/protein components of NETs on T cells. These results implied that TPCN therapy can also maintain immune homeostasis by regulating NET-mediated Treg/Th17 cell imbalance.

In summary, our findings demonstrated nanotherapy-mediated site-specific attenuation of oxidative stress and inflammatory responses in neutrophils is a promising strategy for effective treatment of neutrophilic asthma, by precisely inhibiting NETosis and promoting immune homeostasis. Our results also suggested that the ROS-NETs-Treg/Th17 axis may be potentially new therapeutic targets for severe asthma and other neutrophilic inflammation-associated noninfectious and infectious lung diseases such as adult respiratory distress syndrome, chronic obstructive pulmonary disease, cystic fibrosis, and coronavirus disease (COVID-19), in view of the important pathological effects of neutrophils.

\section{Online content}

Any methods, Nature Research reporting summaries, source data, Supplementary, supplementary information, acknowledgements; details of author contributions and competing interests; and statements of data and code availability are available. 


\section{References}

1. Holgate, S.T. et al. Asthma. Nat. Rev. Dis. Primers.1, 15025 (2015).

2. Soriano, J.B. et al. Prevalence and attributable health burden of chronic respiratory diseases, 19902017: A systematic analysis for the global burden of disease study 2017. Lancet Respir. Med.8, 585596 (2020).

3. James, S.L. et al. Global, regional, and national incidence, prevalence, and years lived with disability for 354 diseases and injuries for 195 countries and territories, 1990-2017: A systematic analysis for the global burden of disease study 2017. Lancet392, 1789-1858 (2018).

4. De Volder, J., Vereecke, L., Joos, G. \& Maes, T. Targeting neutrophils in asthma: A therapeutic opportunity? Biochem. Pharmacol.182, 114292 (2020).

5. https://ginasthma.org/wp-content/uploads/2020/2006/GINA-2020-report_2020_2006_2004-2021wms.pdf

6. Foster, J.M., McDonald, V.M., Guo, M. \& Reddel, H.K. "I have lost in every facet of my life": The hidden burden of severe asthma. Eur. Resp. J.50, 1700765 (2017).

7. Lefebvre, P. et al. Acute and chronic systemic corticosteroid-related complications in patients with severe asthma. J. Allergy Clin. Immunol.136, 1488-1495 (2015).

8. Maneechotesuwan, K., Essilfie-Quaye, S., Kharitonov, S.A., Adcock, I.M. \& Barnes, P.J. Loss of control of asthma following inhaled corticosteroid withdrawal is associated with increased sputum interleukin-8 and neutrophils. Chest132, 98-105 (2007).

9. Fukakusa, M. et al. Oral corticosteroids decrease eosinophil and cc chemokine expression but increase neutrophil, il-8, and ifn-gamma-inducible protein 10 expression in asthmatic airway mucosa. J. Allergy Clin. Immunol.115, 280-286 (2005).

10. Cox, G. Glucocorticoid treatment inhibits apoptosis in human neutrophils. Separation of survival and activation outcomes. J. Immunol.154, 4719-4725 (1995).

11. Ray, A. \& Kolls, J.K. Neutrophilic inflammation in asthma and association with disease severity. Trends Immunol.38, 942-954 (2017).

12. Ray, A., Raundhal, M., Oriss, T.B., Ray, P. \& Wenzel, S.E. Current concepts of severe asthma. J. Clin. Invest.126, 2394-2403 (2016).

13. Radermecker, C. et al. Locally instructed cxcr4(hi) neutrophils trigger environment-driven allergic asthma through the release of neutrophil extracellular traps. Nat. Immunol.20, 1444-1455 (2019).

14. Krishnamoorthy, N. et al. Neutrophil cytoplasts induce th17 differentiation and skew inflammation toward neutrophilia in severe asthma. Sci. Immunol.3, eaao4747 (2018).

15. Toussaint, M. et al. Host DNA released by netosis promotes rhinovirus-induced type-2 allergic asthma exacerbation. Nat. Med.23, 681-691 (2017).

16. Papayannopoulos, V. Neutrophil extracellular traps in immunity and disease. Nat. Rev. Immunol.18, 134-147 (2018). 
17. Jorch, S.K. \& Kubes, P. An emerging role for neutrophil extracellular traps in noninfectious disease. Nat. Med.23, 279-287 (2017).

18. Nemeth, T., Sperandio, M. \& Mocsai, A. Neutrophils as emerging therapeutic targets. Nat. Rev. Drug Discov.19, 253-275 (2020).

19. Li, L. et al. A broad-spectrum ros-eliminating material for prevention of inflammation and druginduced organ toxicity. Adv. Sci.5, 1800781 (2018).

20. Zhao, S. et al. Lipopolysaccharides promote a shift from th2-derived airway eosinophilic inflammation to th17-derived neutrophilic inflammation in an ovalbumin-sensitized murine asthma model. J. Asthma54, 447-455 (2017).

21. Grainge, C.L. \& Davies, D.E. Epithelial injury and repair in airways diseases. Chest144, 1906-1912 (2013).

22. Georas, S.N. \& Rezaee, F. Epithelial barrier function: At the front line of asthma immunology and allergic airway inflammation. J. Allergy Clin. Immunol.134, 509-520 (2014).

23. Davies, D.E. The role of the epithelium in airway remodeling in asthma. Proc. Am. Thorac. Soc.6, 678682 (2009).

24. Kuzmov, A. \& Minko, T. Nanotechnology approaches for inhalation treatment of lung diseases. J. Control. Release219, 500-518 (2015).

25. Barnes, P.J. The cytokine network in asthma and chronic obstructive pulmonary disease. J. Clin. Invest.118, 3546-3556 (2008).

26. Marrache, S. \& Dhar, S. Biodegradable synthetic high-density lipoprotein nanoparticles for atherosclerosis. Proc. Natl. Acad. Sci. U. S. A.110, 9445-9450 (2013).

27. Thiam, H.R., Wong, S.L., Wagner, D.D. \& Waterman, C.M. Cellular mechanisms of netosis. Annu. Rev. Cell Dev. Biol.36, 191-218 (2020).

28. Busse, W.W. A role for neutrophils in asthma exacerbations. Nat. Med.23, 658-659 (2017).

29. Lloyd, C.M. \& Hawrylowicz, C.M. Regulatory t cells in asthma. Immunity31, 438-449 (2009).

30. Zou, X.L. et al. Th17/treg homeostasis, but not th1/th2 homeostasis, is implicated in exacerbation of human bronchial asthma. Therap. Clin. Risk Manag.14, 1627-1636 (2018).

31. Zhao, J., Lloyd, C.M. \& Noble, A. Th17 responses in chronic allergic airway inflammation abrogate regulatory t-cell-mediated tolerance and contribute to airway remodeling. Mucosal Immunol.6, 335346 (2013).

32. Hekking, P.P. et al. The prevalence of severe refractory asthma. J. Allergy Clin. Immunol.135, 896-902 (2015).

33. Rogliani, P. et al. Severe asthma and biological therapy: When, which, and for whom. Pulm. Ther.6, 47-66 (2020).

34. Hyland, M.E., Masoli, M., Lanario, J.W. \& Jones, R.C. A possible explanation for non-responders, responders and super-responders to biologics in severe asthma. Explor. Res. Hypothesis Med.4, 3538 (2019). 
35. Lachowicz-Scroggins, M.E. et al. Extracellular DNA, neutrophil extracellular traps, and inflammasome activation in severe asthma. Am. J. Respir. Crit. Care Med.199, 1076-1085 (2019).

36. Dworski, R., Simon, H.U., Hoskins, A. \& Yousefi, S. Eosinophil and neutrophil extracellular DNA traps in human allergic asthmatic airways. J. Allergy Clin. Immunol.127, 1260-1266 (2011).

37. Lood, C. et al. Neutrophil extracellular traps enriched in oxidized mitochondrial DNA are interferogenic and contribute to lupus-like disease. Nat. Med.22, 146-153 (2016).

38. Remijsen, Q. et al. Neutrophil extracellular trap cell death requires both autophagy and superoxide generation. Cell Res.21, 290-304 (2011).

39. Choy, D.F. et al. Th2 and th17 inflammatory pathways are reciprocally regulated in asthma. Sci. Transl. Med.7, 301ra129 (2015).

40. Newcomb, D.C. \& Peebles, R.S. Th17-mediated inflammation in asthma. Curr. Opin. Immunol.25, 755760 (2013).

41. Vignali, D.A., Collison, L.W. \& Workman, C.J. How regulatory t cells work. Nat. Rev. Immunol.8, 523532 (2008).

42. Rivas, N.M. \& Chatila, T.A. Regulatory t cells in allergic diseases. J. Allergy Clin. Immunol.138, 639652 (2016).

43. Robinson, D.S. Regulatory t cells and asthma. Clin. Exp. Allergy39, 1314-1323 (2009).

44. Zhu, J. et al. Altered expression of regulatory $t$ and th17 cells in murine bronchial asthma. Exp. Ther. Med.14, 714-722 (2017).

45. Wei, Y. et al. Regulation of th17/treg function contributes to the attenuation of chronic airway inflammation by icariin in ovalbumin-induced murine asthma model. Immunobiology220, 789-797 (2015).

46. Chesné, J. et al. II-17 in severe asthma. Where do we stand? Am. J. Resp. Crit. Care.190, 1094-1101 (2014).

\section{Figures}



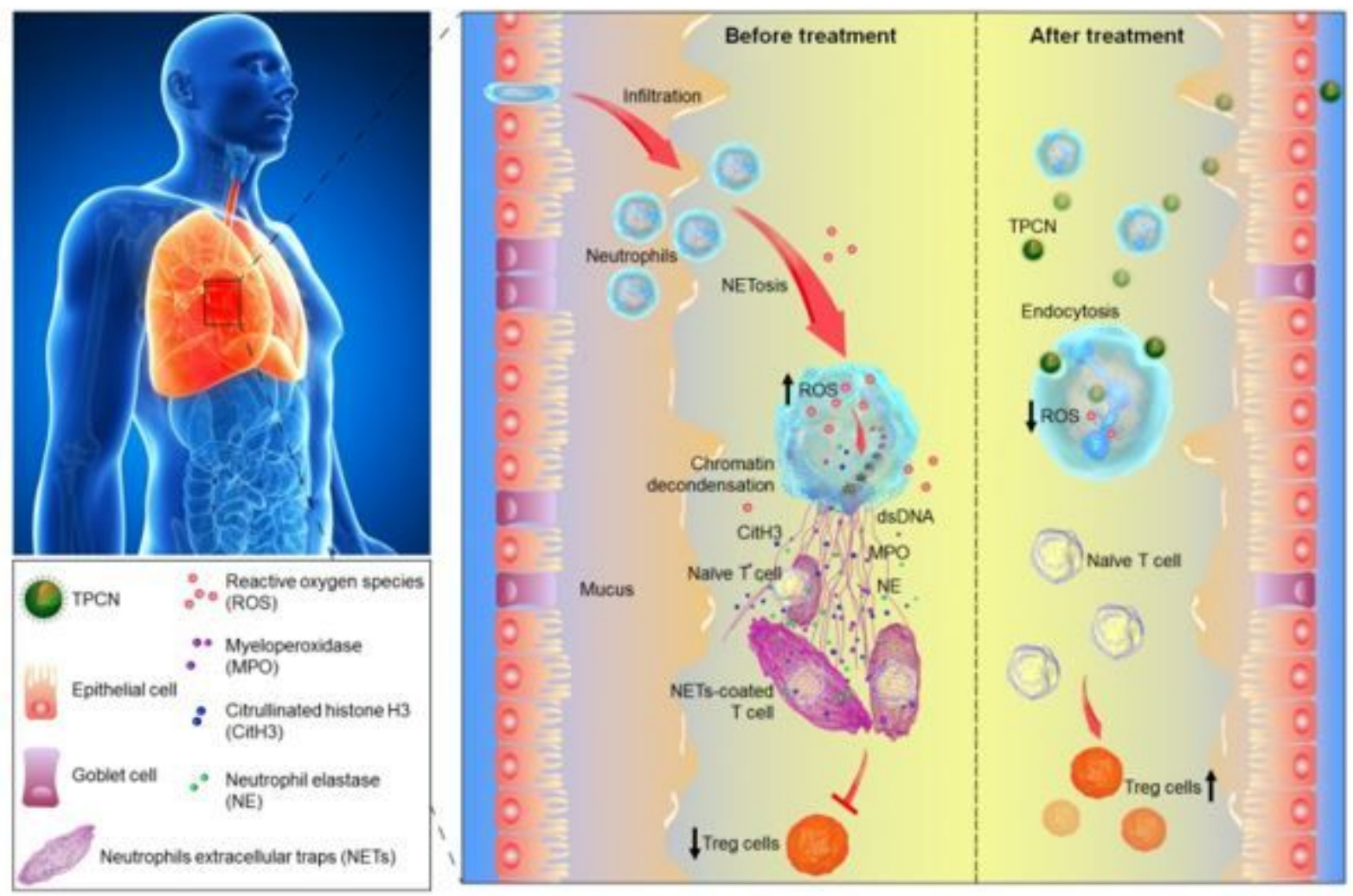

b
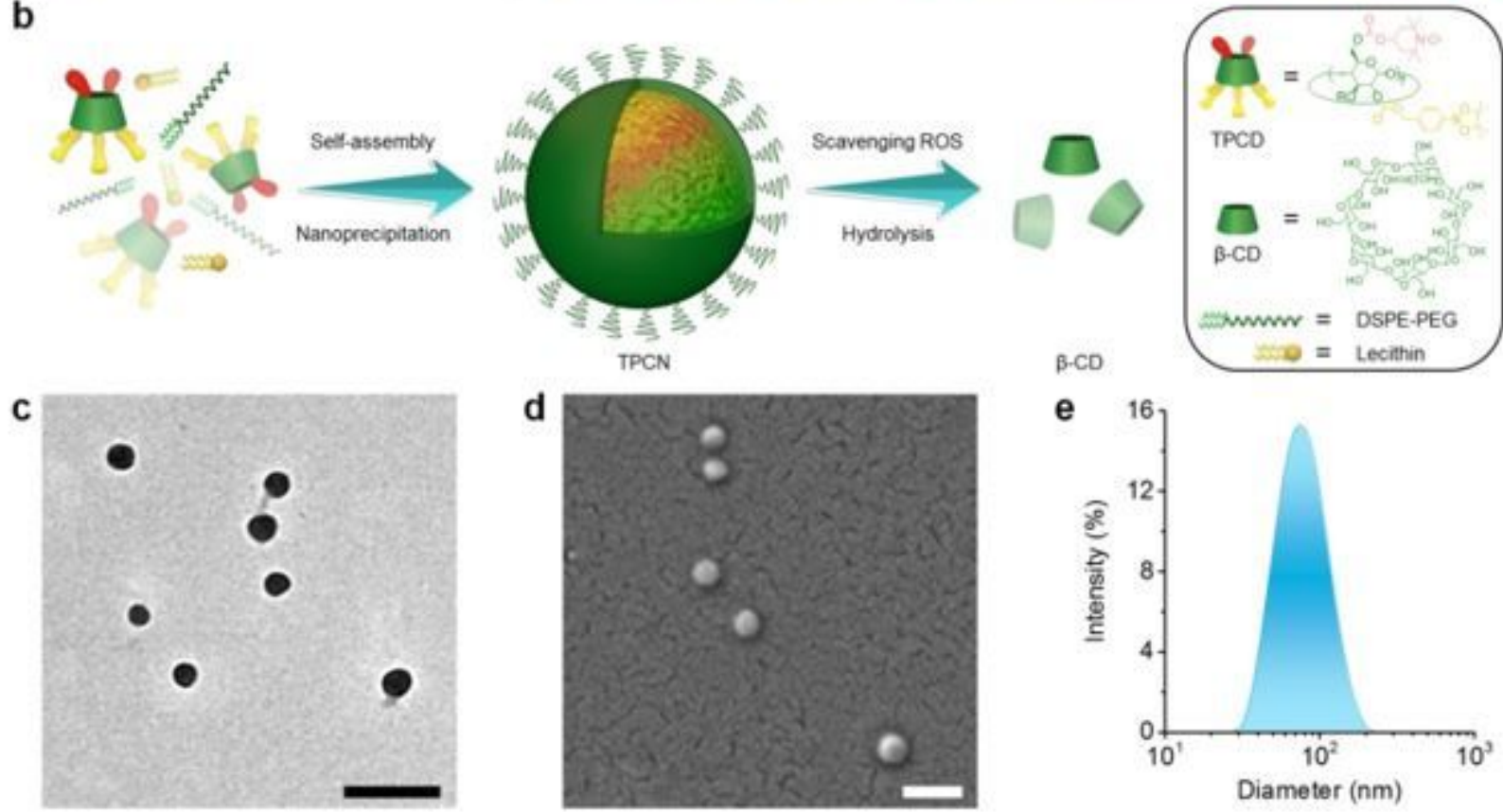

\section{Figure 1}

Schematic illustration of precision therapy of asthma by site-specific inhibition of NETosis and engineering of an antioxidative nanotherapy. a, A sketch showing site-specific elimination of oxidative stress in neutrophils by a nanotherapy TPCN for targeted asthma therapy via regulating neutrophil extracellular trap (NET)-mediated Treg/Th17 cell imbalance. b, Engineering of an antioxidative and anti- 
inflammatory nanotherapy TPCN. c-e, TEM (c) and SEM (d) micrographs as well as size distribution (e) of the nanotherapy TPCN. Scale bars, $200 \mathrm{~nm}$.
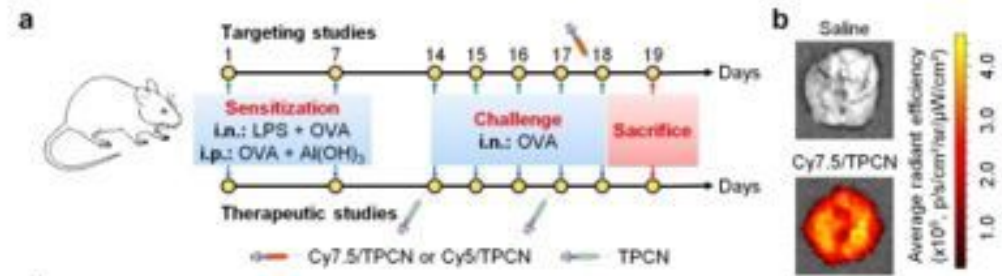

d
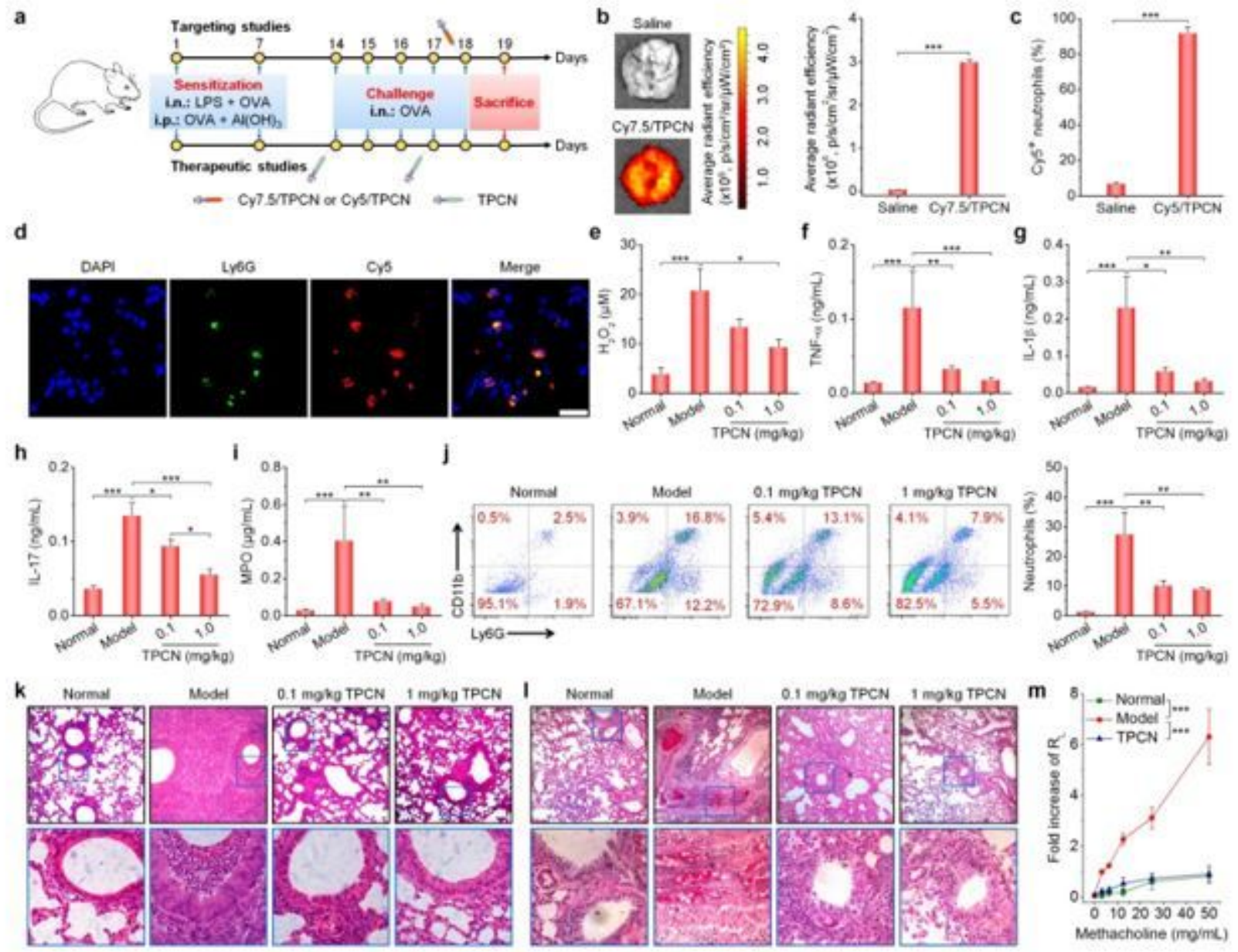

\section{Figure 2}

Targeted therapy of asthma in mice via i.v. injection of TPCN. a, Schematic of the establishment of a mouse model of neutrophilic asthma and regimens for targeting and therapeutic studies. b, Ex vivo images (left) and quantitative analysis (right) showing the accumulation of Cy7.5/TPCN in lungs of asthmatic mice at $24 \mathrm{~h}$ after i.v. administration. c, Flow cytometric quantification of Cy5-positive neutrophils in lung tissues isolated from asthmatic mice at $24 \mathrm{~h}$ after i.v. injection of Cy5/TPCN. d, Representative confocal images illustrate co-localization of Cy5/TPCN (red) with neutrophils (green) in lung tissues excised from asthmatic mice at $24 \mathrm{~h}$ after i.v. injection. Scale bar, $20 \mu \mathrm{m}$. e-i, The levels of

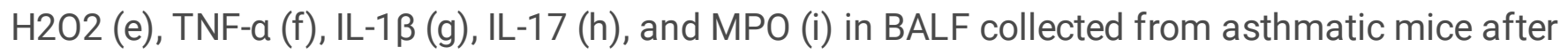
treatment with different formulations. j, Representative flow cytometric profiles (left) and quantitative analysis (right) of neutrophils in BALF of asthmatic mice subjected to different treatments. $\mathrm{k}-\mathrm{I}, \mathrm{H} \& \mathrm{E}(\mathrm{k})$ and PAS ( $($ ) stained histological sections of lung tissues. $m$, Airway hyperresponsiveness of normal or asthmatic mice to aerosolized methacholine. Data are mean \pm s.e.m. $(b, m, n=3 ; c, n=5 ; e-j, n=6)$. Statistical significance was performed by independent-samples t-test for data in $(b, c)$, one-way ANOVA with post-hoc LSD tests for data in (e-j,m). ${ }^{\star} P<0.05$, ${ }^{\star *} P<0.01$, ${ }^{\star * * P}<0.001$. 

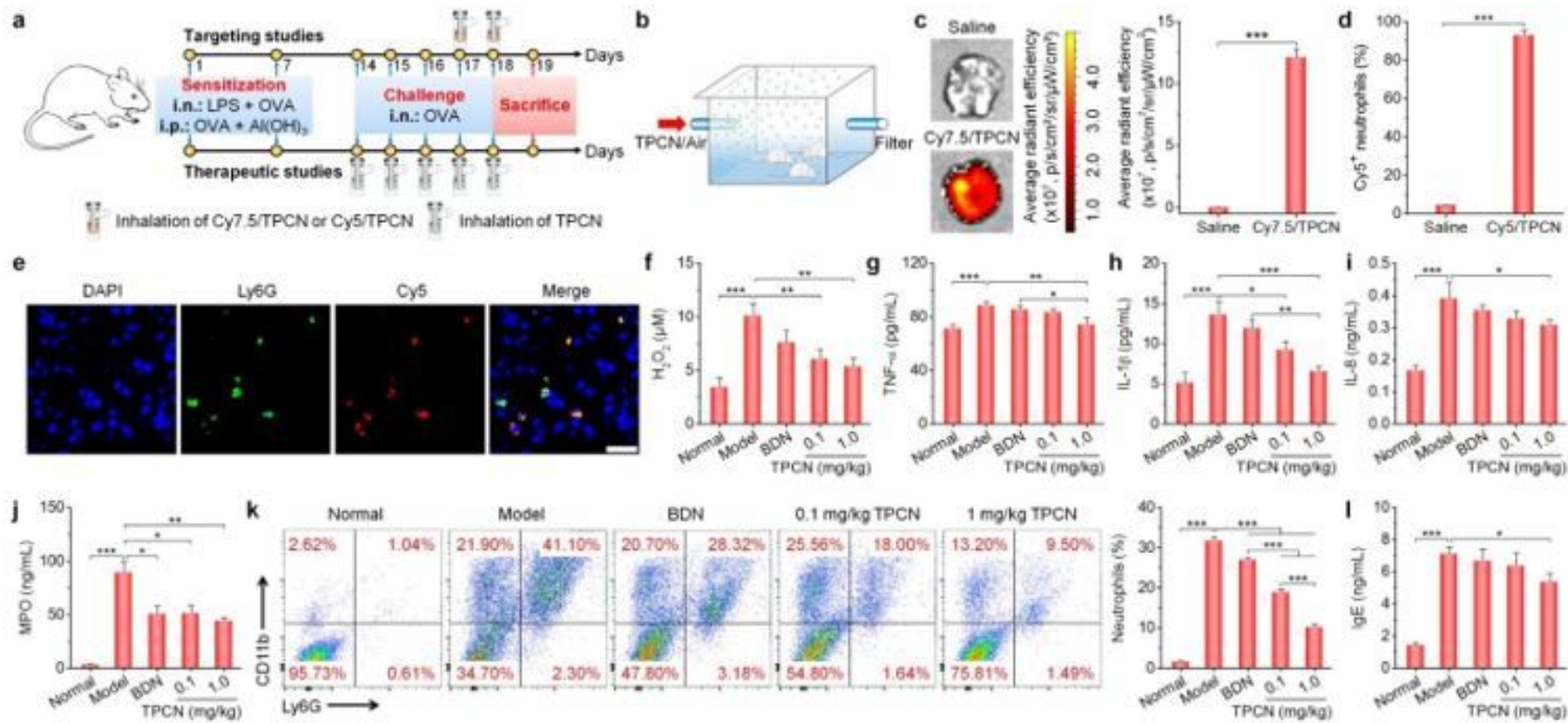

Model BDN
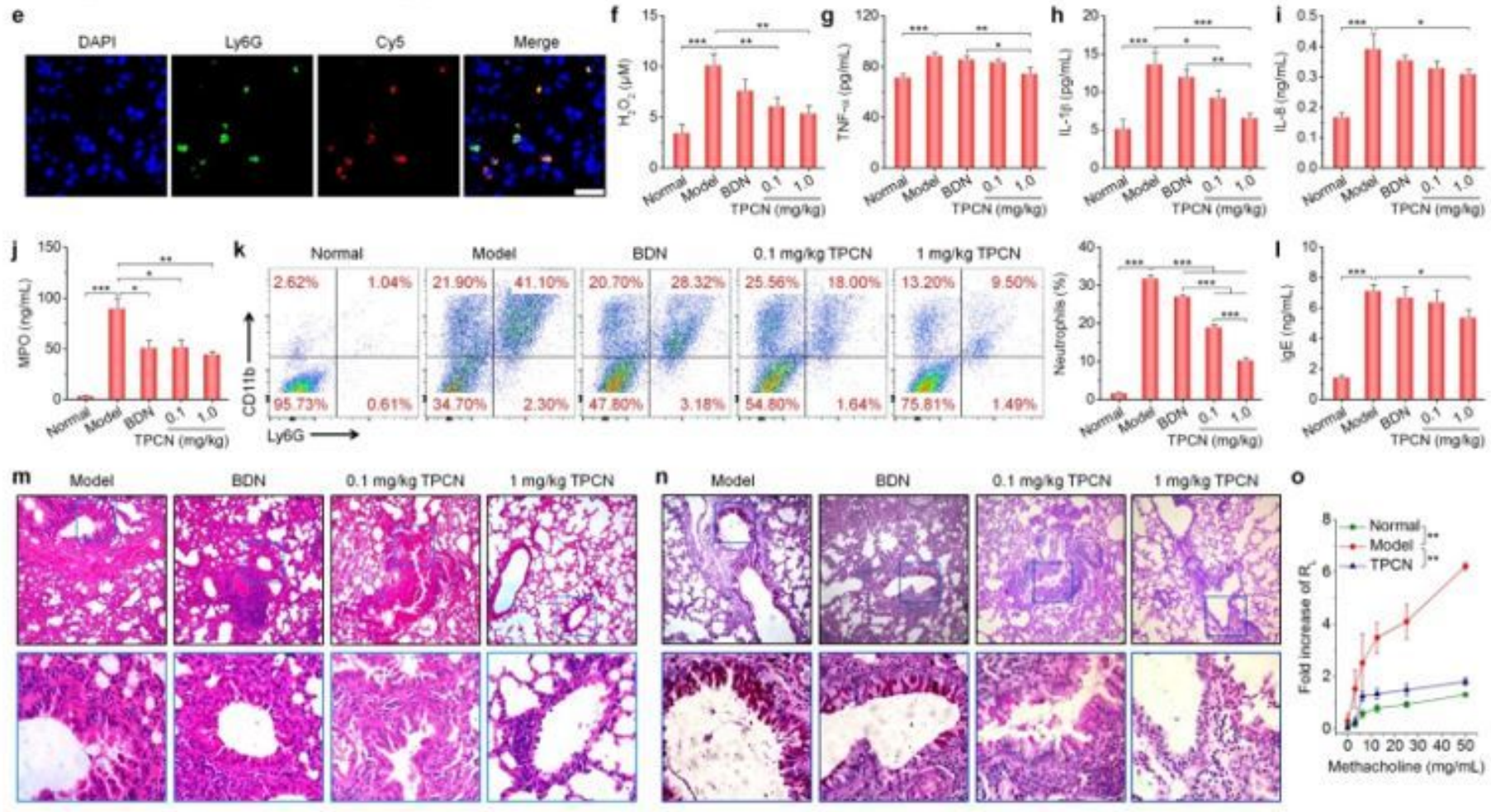

\section{Figure 3}

Targeted therapy of asthma in mice by aerosol inhalation of TPCN. a, Schematic illustration of procedures for targeting and therapeutic studies via aerosol inhalation. b, A sketch showing a platform for aerosolized drug delivery. C, Ex vivo images (left) and quantitative analysis (right) of Cy7.5/TPCN accumulation in lungs of asthmatic mice at $24 \mathrm{~h}$ after aerosol inhalation. $\mathrm{d}$, Quantification of Cy5positive neutrophils in lung tissues of asthmatic mice at $24 \mathrm{~h}$ after inhalation. e, Fluorescence images of cryosections of lung tissues from asthmatic mice at $24 \mathrm{~h}$ after aerosol inhalation of Cy5/TPCN. Scale bar, $20 \mu \mathrm{m}$. f-j, The expression levels of H2O2 (f), TNF-a (g), IL-1 $\beta$ (h), IL-8 (i), and MPO (j) in BALF from asthmatic mice after inhalation of different formulations. $k$, Flow cytometric quantification of neutrophils in BALF. I, Serum IgE levels from asthma mice subjected to different treatments. $m-n, H \& E(m)$ and PAS (n) stained pathological sections of lung tissues. o, Invasive measurement of airway hyperresponsiveness of normal or asthmatic mice to challenge with methacholine aerosol. Data are mean \pm s.e.m. $(c, o, n=3 ; d, n=5 ; f-l, n=6)$. Statistical significance was performed by independentsamples t-test for data in (c,d) and by one-way ANOVA with post-hoc LSD tests for data in $(\mathrm{f}-\mathrm{l}, \mathrm{o})$. ${ }^{*} \mathrm{P}<$ $0.05, * \star P<0.01, * \star * P<0.001$. 

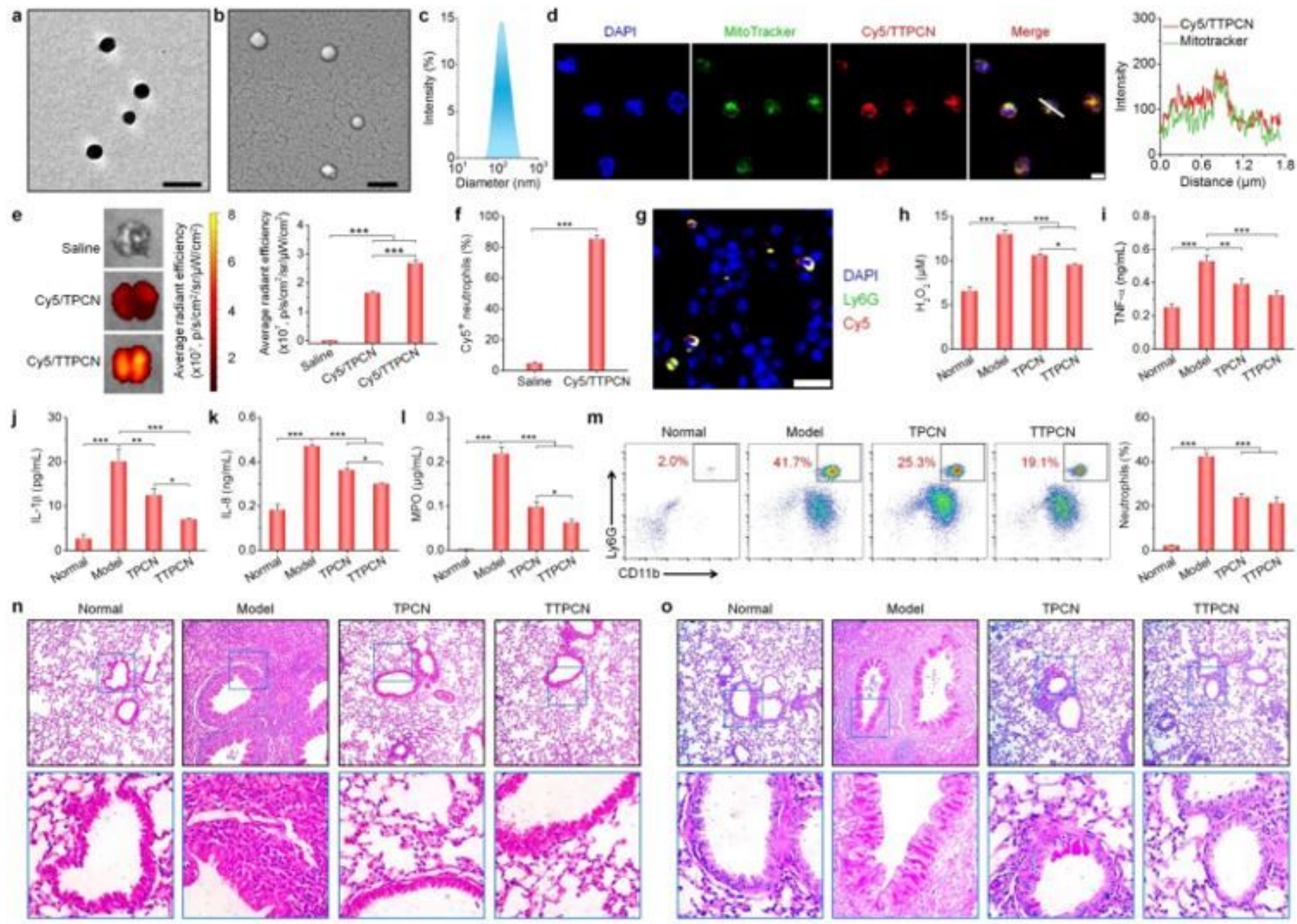

\section{Figure 4}

Therapy of neutrophilic asthma in mice with a mitochondrial targeting nanotherapy TTPCN after i.v. injection. a-c, SEM (a) and TEM (b) images as well as size distribution (c) of TTPCN. Scale bars, $200 \mathrm{~nm}$. d, Confocal microscopy images show mitochondrial localization of Cy5-labeled TTPCN in neutrophils. The right panel shows quantitative analysis of fluorescent intensities in a single cell as highlighted by the white line in the merge image. Neutrophils were incubated with targeting TTPCN (red) for $1 \mathrm{~h}$, followed by staining with a mitochondrial marker MitoTracker (green). Scale bar, $5 \mu \mathrm{m}$. e, Ex vivo images (left) and quantitative analysis (right) of fluorescence distribution in lungs of asthmatic mice treated with Cy5/TPCN or Cy5/TTPCN. f, Flow cytometry analysis of Cy5-positive neutrophils in lung tissues from asthma mice at $24 \mathrm{~h}$ after i.v. injection of Cy5/TTPCN. g, A representative fluorescence image shows colocalization of Cy5/TTPCN with Ly6G-positive neutrophils. Scale bar, $20 \mu \mathrm{m}$. h-l, Levels of H2O2 (h), TNF$a(i), I L-1 \beta(j), I L-8(k)$, and MPO (I) in BALF from healthy or asthma mice subjected to different treatments. $\mathrm{m}$, Flow cytometric profiles (left) and quantitative data (right) illustrating neutrophil counts in BALF from mice. $n-0, H \& E(n)$ and PAS (o) stained pathological sections of lung tissues. Data are mean $\pm S E(e, n=3$; $f, h-m, n=5)$. Statistical analysis was conducted by one-way ANOVA with post-hoc LSD tests for data in 
(e,h-j,l-m), by the independent-samples t-test for data in (f), and by the Kruskal-Wallis H test for data in (k). *P $<0.05$, ** $P<0.01$, *** $P<0.001$.

a
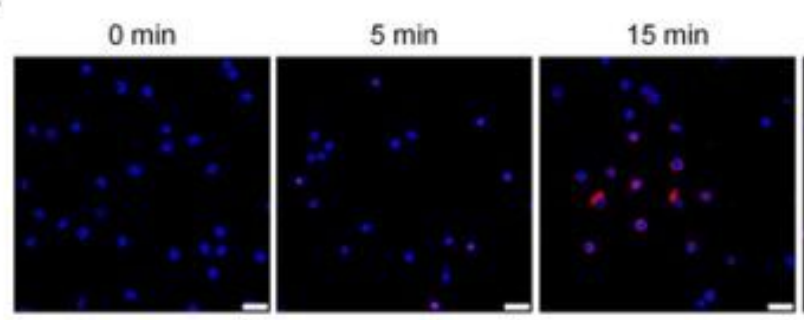

C
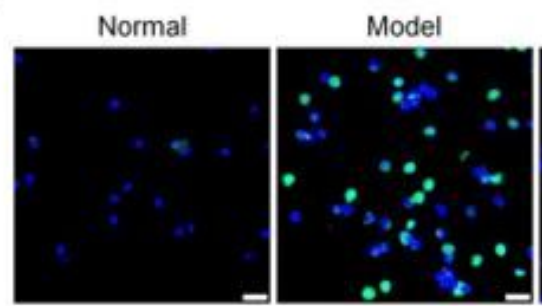

$25 \mu \mathrm{g} / \mathrm{mL}$ TPCN
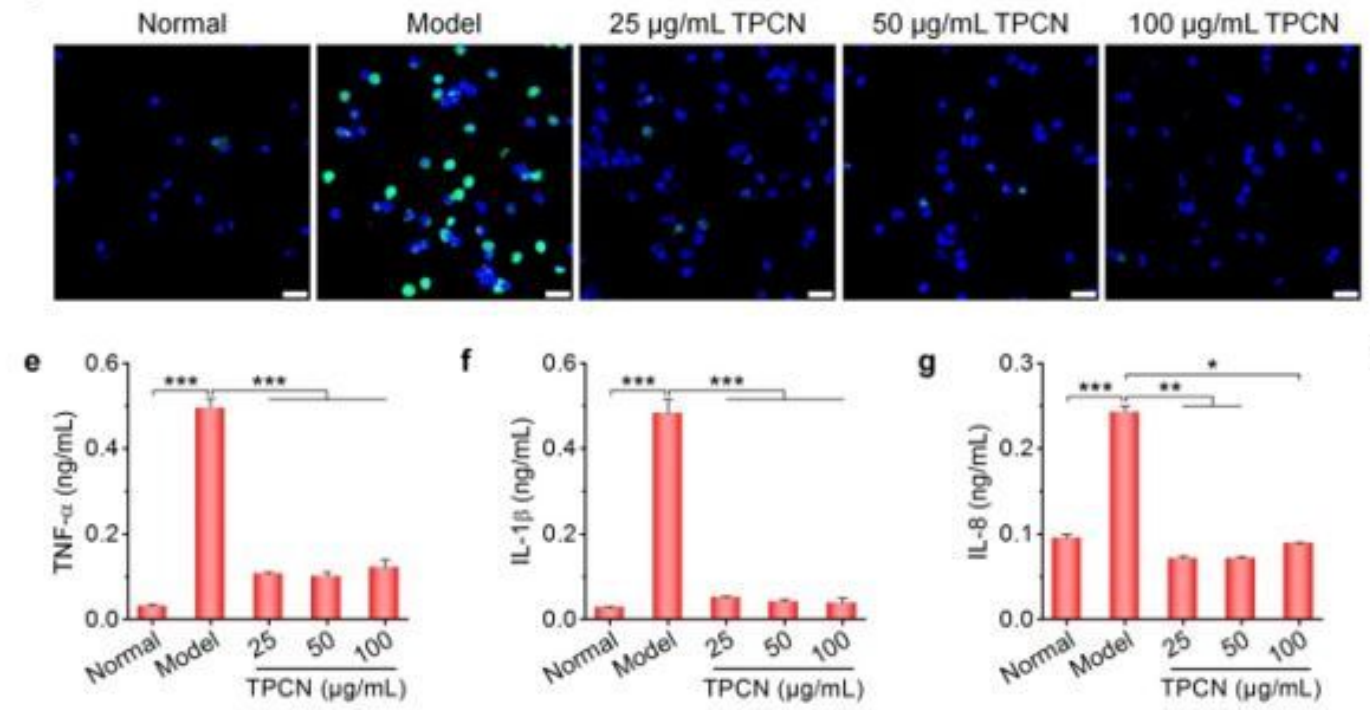

$\mathbf{i}$

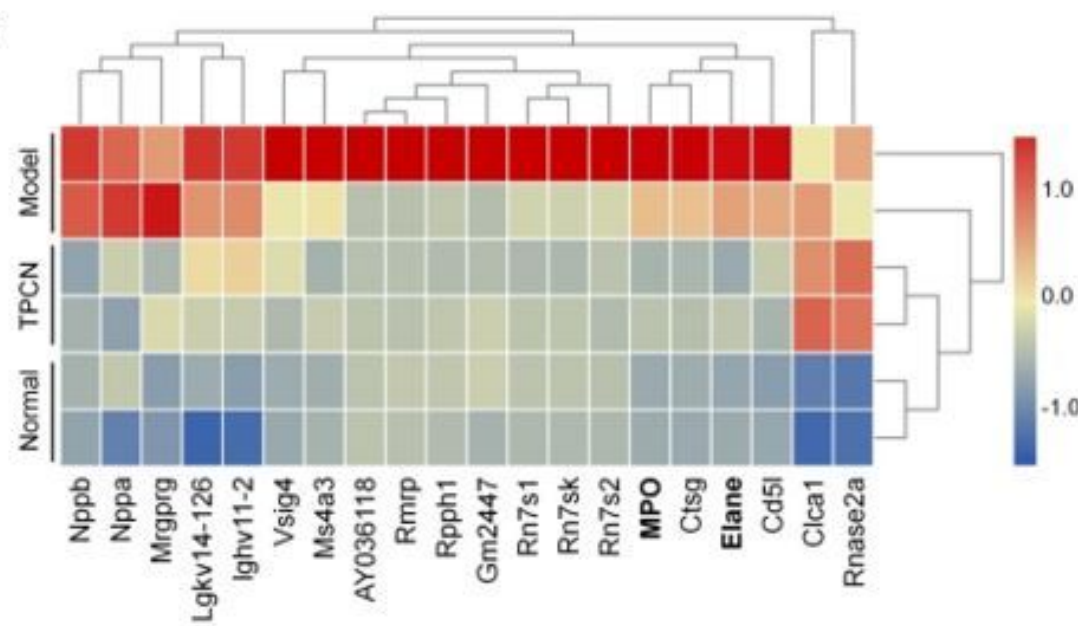

g

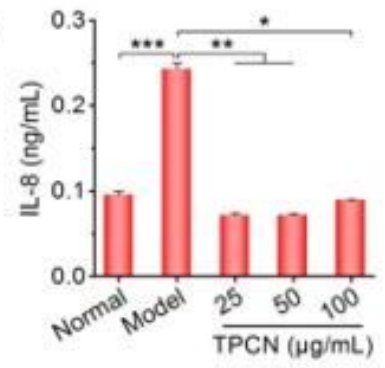

j

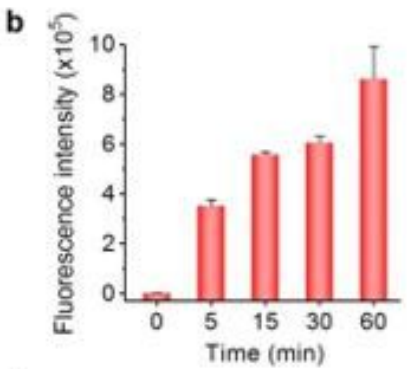

d

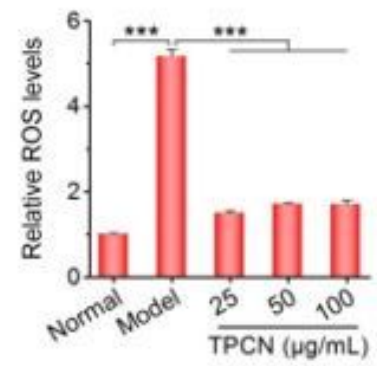

h

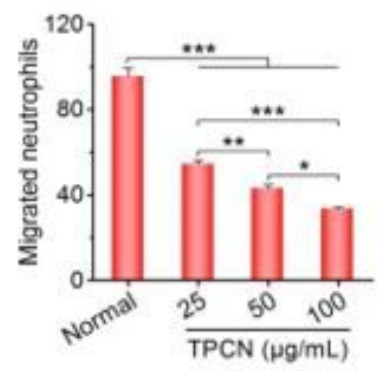

k
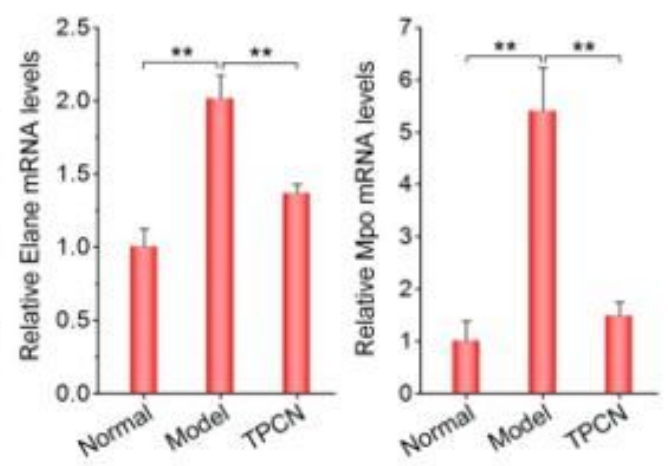

\section{Figure 5}

In vitro bioactive effects of TPCN in neutrophils and RNA-sequencing analysis of pulmonary tissues from healthy or asthmatic mice with or without TPCN treatment. a-b, Fluorescence images (a) and flow cytometry quantification (b) of time-dependent endocytosis of Cy5/TPCN in neutrophils. Nuclei were stained with DAPI (blue). Scale bars, $20 \mu \mathrm{m}$. c, Fluorescence images indicate ROS production in neutrophils after different treatments and staining with a fluorescent probe DCFH-DA. The normal group was treated with fresh medium, while the model group was induced with PMA. Before stimulation with 
PMA, the TPCN groups were treated with TPCN at varied doses. Scale bars, $20 \mu \mathrm{m}$. $d$, Relative ROS levels in neutrophils quantified by flow cytometry after staining with DCFH-DA. e-g, Expression levels of TNF-a (e), IL-1 $(\mathrm{f})$, and IL-8 $(\mathrm{g})$ in neutrophils after different treatments. $h$, TPCN inhibits in vitro migration of peritoneal neutrophils. i, Clustering analysis showing top 20 regulated genes in lungs of healthy or asthmatic mice after different treatments. j-k, Elane and Mpo mRNA levels quantified by qRT-PCR. Data are mean \pm s.e.m. ( $(b, n=6 ; d, n=4 ; e-h, n=5 ; j-k, n=3)$. Statistical analysis was conducted by one-way ANOVA with post-hoc LSD tests. ${ }^{*} \mathrm{P}<0.05, * \star \mathrm{P}<0.01$, $* \star * P<0.001$.
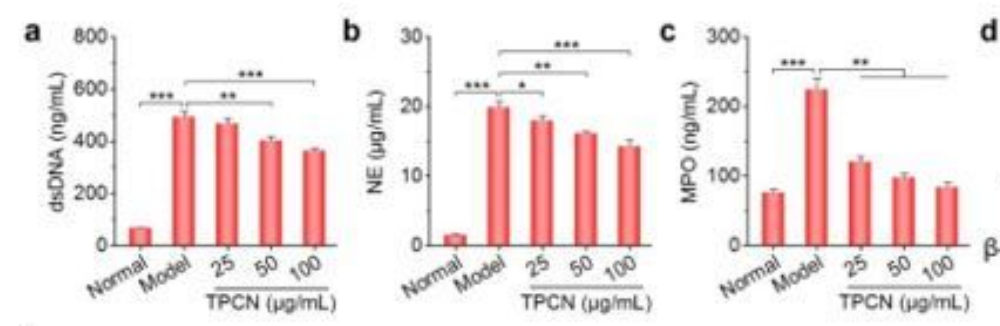

d
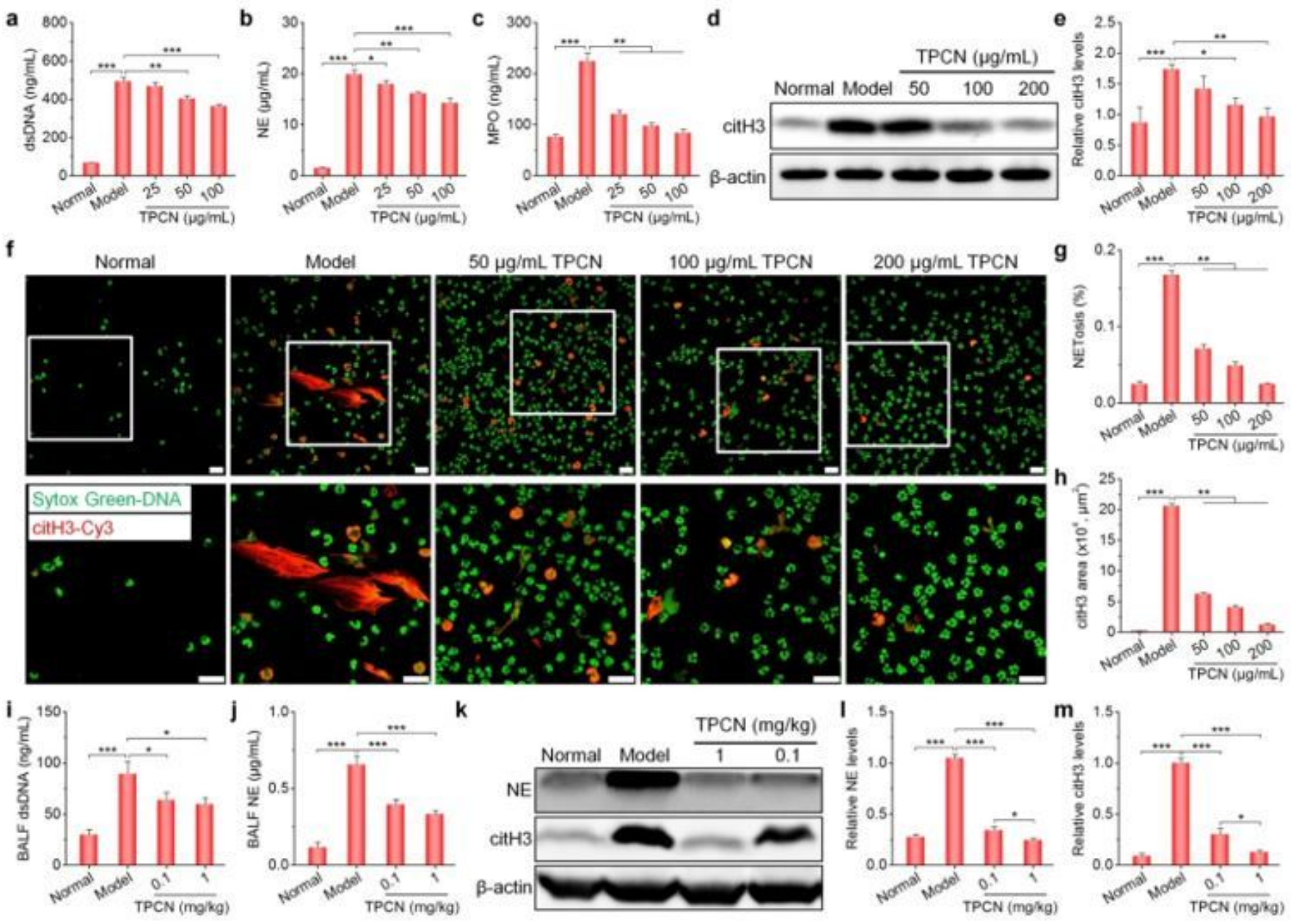

n

Normal

Model

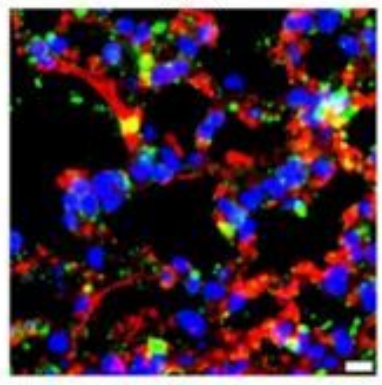

$0.1 \mathrm{mg} / \mathrm{kg}$ TPCN

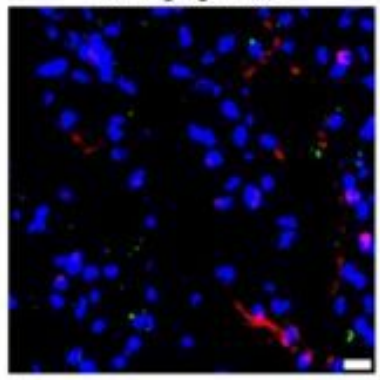

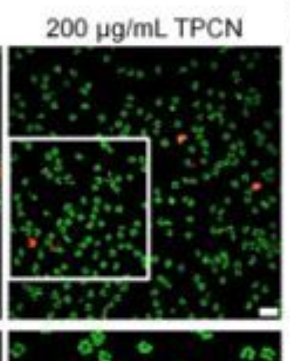
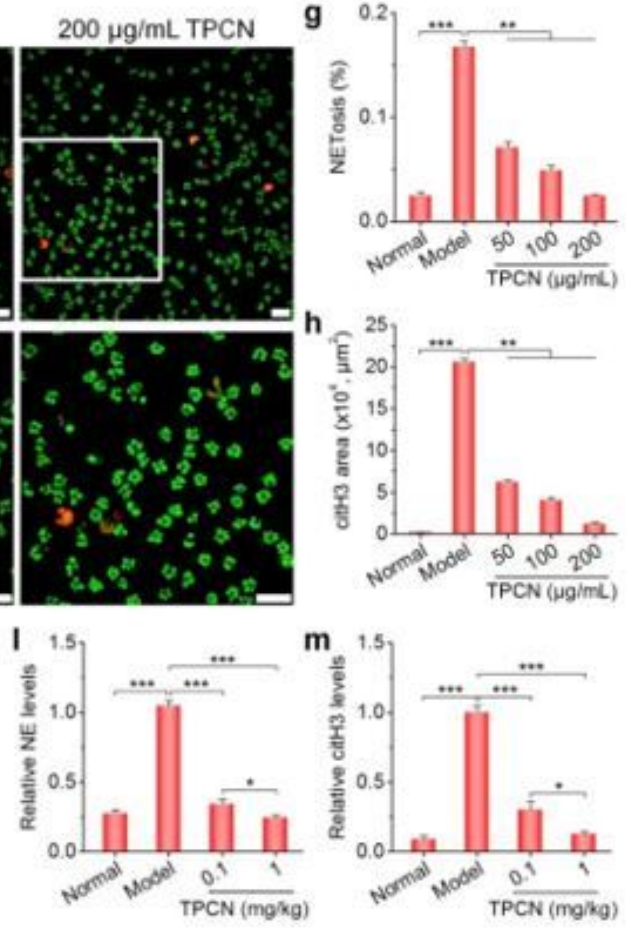

$1 \mathrm{mg} / \mathrm{kg}$ TPCN

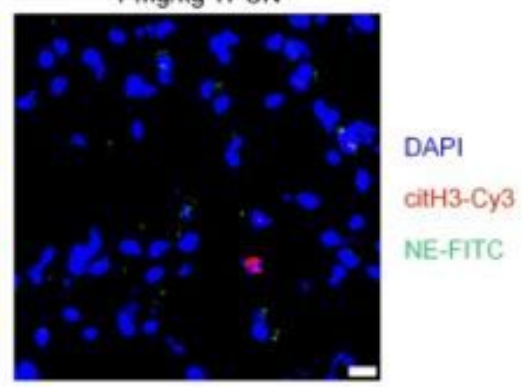

\section{Figure 6}

TPCN inhibits NETosis in vitro and in vivo. a-c, Quantified levels of extracellular dsDNA (a), neutrophil elastase (NE) (b), and MPO (c). d-e, Representative Western blotting bands (d) and normalized levels (e) 
of citrullinated histone $\mathrm{H} 3$ (citH3) in the acellular fraction. In all cases, peritoneal neutrophils treated with fresh medium served as the normal group, while neutrophils in the model group were stimulated with PMA. TPCN groups were incubated with PMA and various doses of TPCN. f, Confocal microscopy observation of the NETs formation in neutrophils after different treatments, as indicated by colocalization of extracellular dsDNA and citH3. Scale bars, $20 \mu \mathrm{m}$. g-h, Quantification of the NETosis degree (g) and citH3 area (h). i-j, Quantified levels of extracellular dsDNA (i) and NE (j) in BALF of mice treated with different formulations. $k-m$, Representative bands $(k)$ and quantitative analysis of NE $(I)$ and citH3 (m) in lung tissue protein extracts. n, Immunofluorescence analysis of citH3 and NE in lung sections of mice subjected to different treatments. Scale bars, $20 \mu \mathrm{m}$. Data are mean \pm s.e.m. $(a-c, i-j, n=5 ;$ e,l-m, $n$ $=3 ; g-h, n=4)$. Statistical analysis was conducted by one-way ANOVA with post-hoc LSD tests. ${ }^{*}<0.05$, $\star \star P<0.01, * \star * P<0.001$. 
a
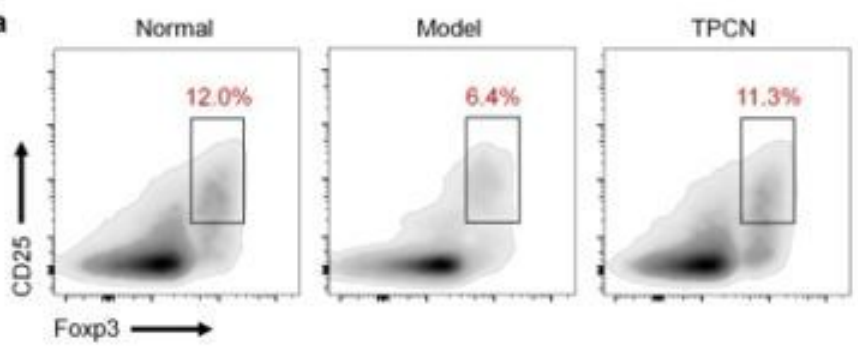

b
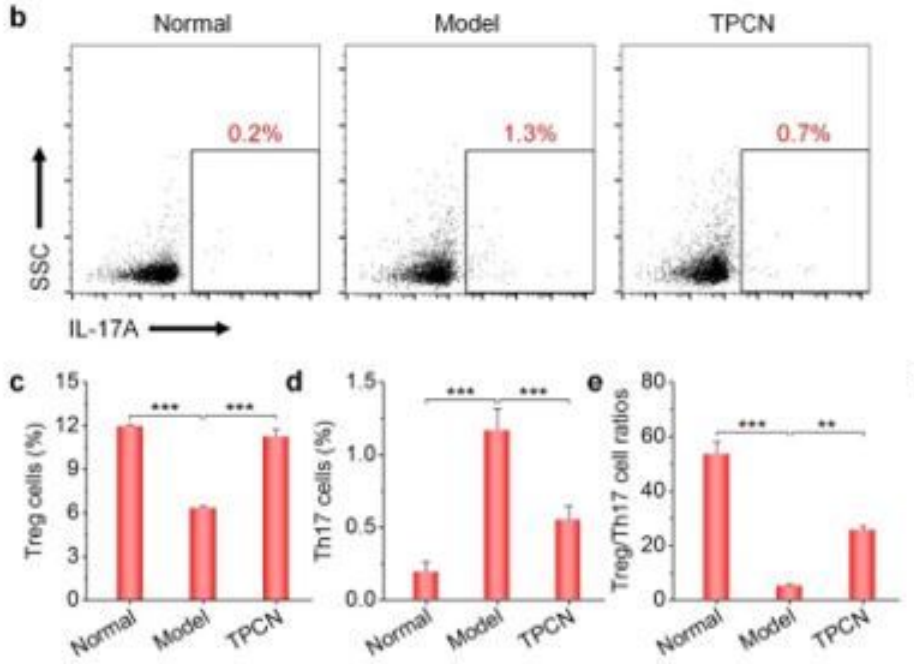

k

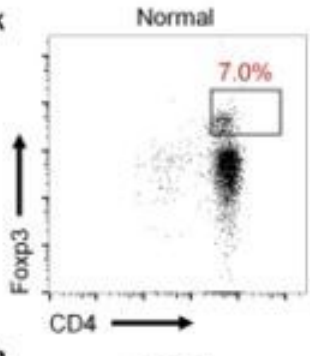

Control

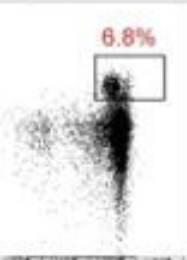

Model

n

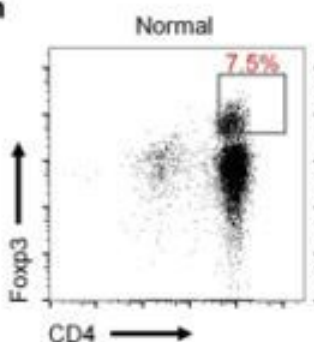

$\circ$

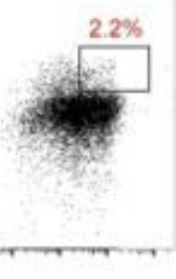

f
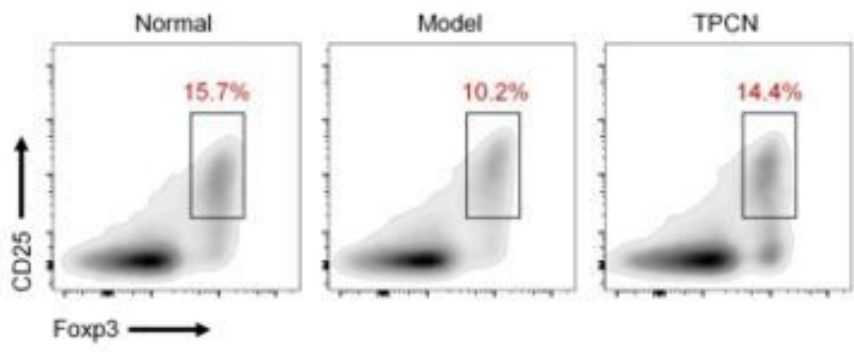

g
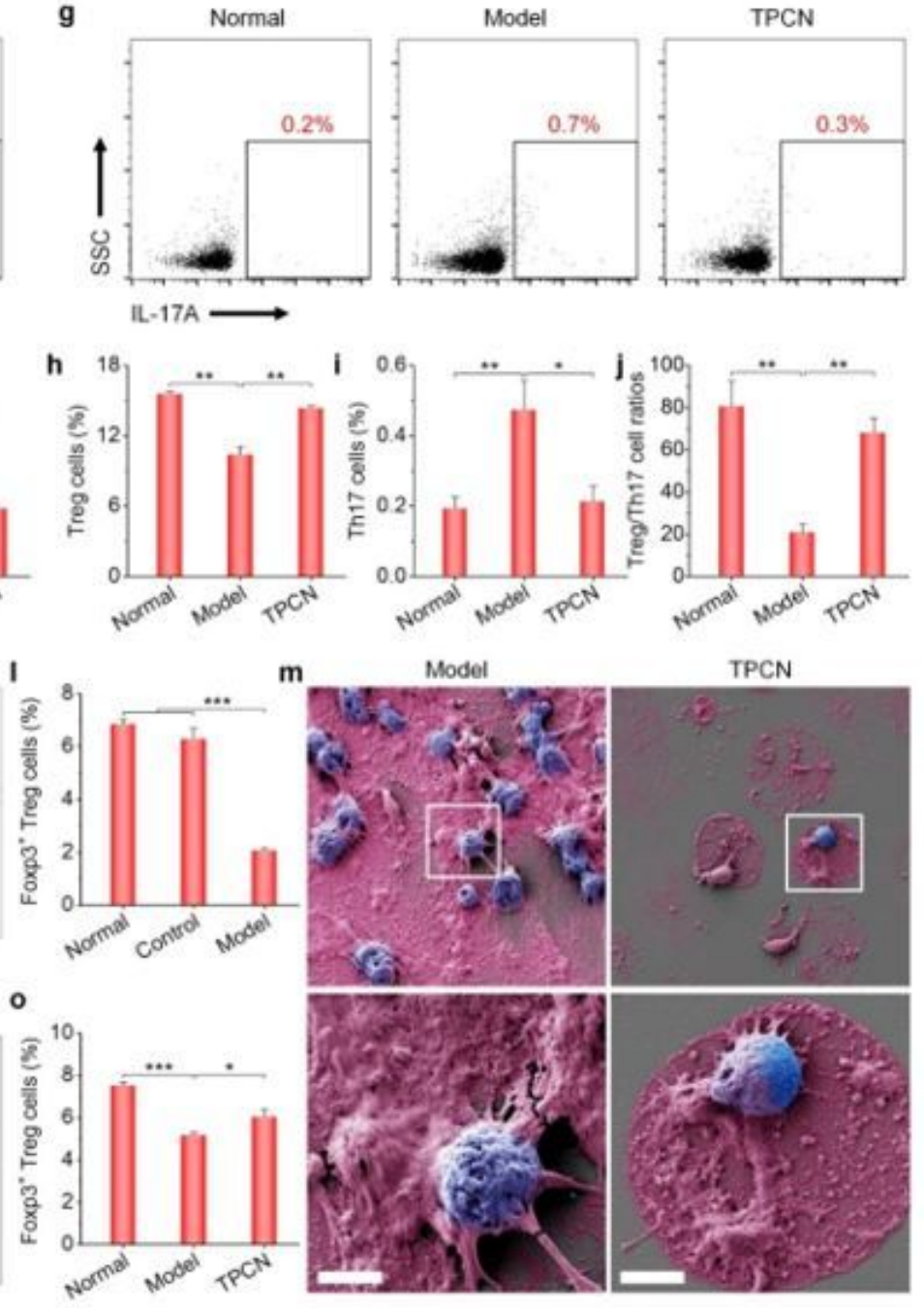

\section{Figure 7}

The effects of TPCN on NETs-mediated differention of regulatory T cells. a-e, Representative flow cytomeitric profiles (a-b), quantiative analysis of Treg and Th17 cells (c-d), and Treg/Th17 cell count ratios (e) in lung tissues of healthy or asthmatic mice with or without TPCN treatment. f-j, Flow cytometric profiles (f-g), quantification of Treg and Th17 cells (h-i), and calculation of Treg/Th17 cell count ratios (j) in splenic tissues from healthy or asthmatic mice after different treatments. k-I, Flow cytometric profiles (k) and quantitative analysis (I) of in vitro Treg cell differentiation in the presence of neutrophils (control) or NETs (model). Naïve T cells in the normal group were treated with medium alone. $\mathrm{m}$, SEM images show attenuated NETs coating on naïve T cells by TPCN. Non-trapped T cells were largely removed 
during washing procedures. Scale bars, $3 \mu \mathrm{m}$. n-o, Flow cytometric analysis illustrating promoted in vitro Treg cell differentiation by TPCN via inhibiting the NETs formation. Date are mean \pm s.e.m. $(n=4)$.

Statistical analysis was performed by one-way ANOVA with post-hoc LSD tests. ${ }^{*} P<0.05,{ }^{\star * P}<0.01, * \star \star P$ $<0.001$.

\section{Supplementary Files}

This is a list of supplementary files associated with this preprint. Click to download.

- SupplementaryInformationFinal.docx 